\title{
Early Herschel results on evolved stars and planetary nebulae
}

\author{
Pedro García-Lario \\ Herschel Science Centre, European Space Astronomy Centre, European Space Agency, \\ Postbus 78, E-28691 Villanueva de la Cañada, Madrid, Spain \\ email: Pedro.Garcia.Lario@esa.int
}

\begin{abstract}
Herschel, an ESA space observatory equipped with science instruments provided by European-led Principal Investigator consortia with important participation from NASA, was launched on 14 May 2009. With its $3.5 \mathrm{~m}$ diameter primary mirror, Herschel is the largest space telescope ever launched into space, and carries onboard three science instruments, whose focal plane units are cryogenically cooled inside a superfluid helium cryostat. The PACS and SPIRE instruments provide broadband imaging photometry in six bands centred at 75,100 , $160,250,350$, and 500 microns and imaging spectroscopy over the range 55-672 microns. The HIFI instrument provides very high-resolution heterodyne spectroscopy over the ranges 157-212 and 240-625 microns. The results obtained already in the first year and a half of routine science operations demonstrate that Herschel will have strong impact on all research fields, from Solar System studies to the area of Cosmology, from the analysis of star formation to the mysteries of galaxy formation. In this talk I will review the Herschel highlights in the area of evolved stars in general and of planetary nebulae more in particular, resulting from observations performed with the three instruments onboard Herschel since launch. This will be exemplified by a few observational results, just the tip of the iceberg of what is yet to come in the remaining year and a half of science operations.
\end{abstract}

Keywords. Infrared: general, infrared: stars, stars: AGB and post-AGB

\section{Introduction}

ESA's Herschel space observatory (Pilbratt et al. 2010) was successfully launched, together with the Planck satellite, on board an Ariane 5 ECA launcher from Kourou, the European's Spaceport in French Guiana, on 14 May 2009. Equipped with a passively cooled $3.5 \mathrm{~m}$ primary mirror, Herschel is the largest telescope ever launched into space, orbiting around the second Lagrangian point of the Sun-Earth system (L2), at a mean distance of 1.5 million $\mathrm{km}$ in the anti-Sun direction.

With its ability to observe across the far infrared and sub-millimetre wavelengths (55-672 $\mu \mathrm{m})$, Herschel is bridging the gap between earlier infrared space missions and ground-based facilities observing in the submilimeter range. Designed to observe the 'cool and distant Universe', Herschel's primary science objectives are to:

- study the formation of galaxies in the early Universe and their subsequent evolution;

- investigate the creation of stars and their interaction with the interstellar medium;

- observe the chemical composition of the atmospheres and surfaces of comets, planets and satellites in our Solar System, and

- examine the molecular chemistry of the Universe.

For this, Herschel is equipped with three main instruments: HIFI, PACS and SPIRE, housed in a superfluid helium cryostat. The PACS and SPIRE instruments provide broadband imaging photometry in six bands centred at $75,100,160,250,350$, and $500 \mu \mathrm{m}$ and 
imaging spectroscopy over the range 55-672 $\mu \mathrm{m}$, while HIFI provides very high-resolution heterodyne spectroscopy over the ranges 157-212 and 240-625 $\mu \mathrm{m}$.

Herschel is operated as an observatory facility designed to provide a minimum of 3 years of routine science operations, with an estimated total mission lifetime of 3.5 years. As an observatory, it is available to the worldwide scientific community, with roughly two thirds of the observing time considered 'open time', allocated through standard competitive calls for observing proposals.

\section{Early mission phases}

Exactly one month after the launch, Herschel's crycover was successfully opened and the first light images of an astronomical source (M51) were obtained with the PACS instrument, demonstrating a perfect optical performance, fully according to specifications.

Building on the experience from this PACS 'sneak preview', and making use of time initially allocated to 'thermal stabilization' of the whole spacecraft, all three instruments performed their initial test observations, including SPIRE images of nearby galaxies, HIFI spectroscopy of a star forming region and PACS imaging spectroscopy of a planetary nebula. These very first attempts already provided spectacular data, and were followed by the successful completion of 2 months of 'Commissioning Phase', mainly used for instrument and spacecraft functional tests, plus 3 additional months of 'Performance Verification', when the different planned instrument observing modes were optimised according to the results obtained in-orbit.

As a consequence of the experience gained during this three-month period, various observing modes were declared 'ready to use' in a gradual fashion leading to the start of the so-called 'Science Demonstration Phase', in which small snippets of the various observing programmes were executed for verification that the users were getting what they wanted; otherwise they had to update and optimize their observing programmes.

The first 'Science Demonstration Phase' observations were delivered to users on 28 September 2009 and since then all remaining observing modes were released, adapted to in-flight circumstances. In this process, some observing modes were discarded, while some others had to be fully revamped.

This sliding transition took longer than initially expected, as HIFI became unavailable on 2 August 2009, affected by an anomaly, and was only recovered for science in April 2010. Despite this adversity, we can say that PACS and SPIRE have routinely been operated since mid-December 2009 and HIFI since mid-April 2010. At the present moment, the three instruments are being operated at full speed.

\section{Early science results}

In the first year of Routine Science Phase operations Herschel has started to do the science that it will continue doing for as long as the observatory will work. And from the very beginning Herschel has demonstrated that the scientific results that will be obtained will have an enormous impact on essentially all fields of astronomy, from Solar System studies to Cosmology, from the analysis of star formation, included the origin of our own Sun, to the models which describe the formation of the first galaxies in the very early days of our Universe, including the physical process that take place in the interstellar medium and the feedback material that is returned by evolved stars and make the Universe look the way it looks nowadays.

A nice overview of the initial results obtained by the various Herschel Key Programmes approved for execution in the first year of the mission can be found in e.g. Pilbratt 2011 or García-Lario 2011, and references therein. 


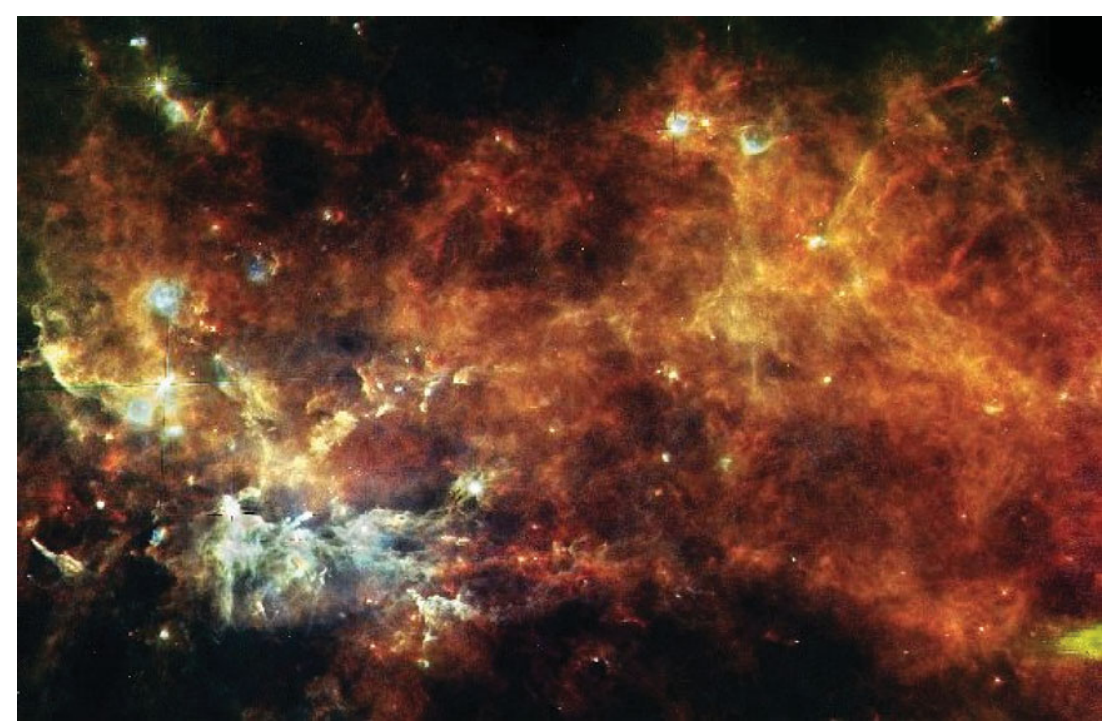

Figure 1. PACS/SPIRE three colour composite image (blue $=70 \mu \mathrm{m}$, green $=160 \mu \mathrm{m}$, red $=$ $350 \mu \mathrm{m}$ ) which unveils our own Milky Way Galaxy around $l=59^{\circ}$ as one giant nursery where generations of new young stars are continuously born (Molinari et al. 2010)

Some of the initial findings have been put together in two special issues of Astronomy \& Astrophysics (vols. 518 and 521). They contain in total more than 200 refereed papers describing just the first 3 months of scientific operations with Herschel, out of which, a total of 21 papers address various aspects related to the physics of evolved stars and their environments.

\section{Evolved stars and planetary nebulae studies with Herschel}

\subsection{Herschel Key Programmes}

MESS and HIFISTARS are the only two Key Programmes approved for execution (out of a total number of 42) covering various aspects of the physics and chemical evolution of Galactic AGB stars and PNe, for a total observing time of 500 hours, representing $10 \%$ of the total time allocated to proposals in this format.

MESS: (Mass Loss in Evolved StarS; P.I.: M. Groenewegen) is mainly devoted to the obtention of PACS and SPIRE imaging and spectroscopy of a large number of stars in various evolutionary stages from the AGB to the PN phase.

As part of the imaging programme, detached shells have been detected around a number of dying stars, like AQ And, U Ant and TT Cyg using PACS photometry at 70, 100 and $160 \mu \mathrm{m}$ (Kerschbaum et al. 2010) and others (Kerschbaum et al. 2011). Wind-ISM interactions of various kinds have been observed in many of the sources targeted by this programme. Bow shocks are reported for CW Leo = IRAC+10216 (Ladjal et al. 2010), X Her and TX Psc (Jorissen et al. 2011), and $\alpha$ Ori (Decin et al. in preparation). For $o$ Cet (Mira) the inner part of the stellar wind bubble bounded and formed by the termination shock is seen (Mayer et al. 2011). Overall considered, bow shocks are observed for $\sim 40 \%$ of the sample and detached rings for $\sim 20 \%$ (Cox et al. 2011). The star's peculiar velocity and the density of the ISM appear decisive in the detection of emission from bow shocks or detached rings. Herschel now reveals these infrared shells and bow shock regions at the best spatial resolution ever. 


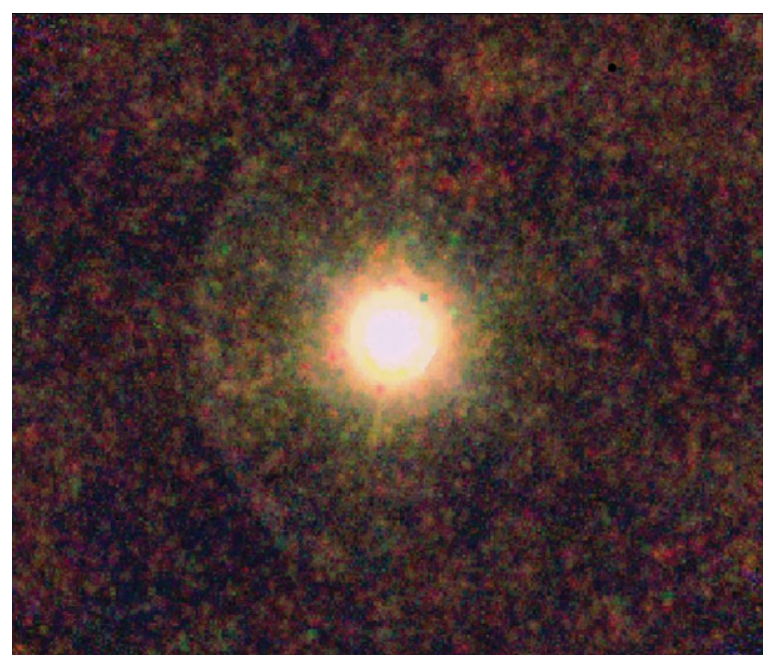

Figure 2. Combined PACS $160 \mu \mathrm{m}$ and SPIRE 250 and $350 \mu \mathrm{m}$ image of CW Leo $=$ IRC +10216 . An incomplete arc of 22' diameter, which is cospatial with the termination shock due to interaction with the ISM observed in UV GALEX images is clearly seen (Ladjal et al. 2010).

Herschel PACS and SPIRE images of NGC $6720=$ the Ring Nebula (van Hoof et al. 2010) and of NGC 7293 = the Helix Nebula (Wesson et al. this proceedings), display a striking resemblance between the far-infrared dust distribution and the $\mathrm{H}_{2}$ emission observed in the near infrared which appears to be observational evidence that $\mathrm{H}_{2}$ forms on grain surfaces.

One of the most interesting spectroscopic results obtained by MESS is the discovery of a multitude (more than 60) of spectral lines from warm water vapour in the circumstellar environment around the ageing carbon star CW Leo $=$ IRC +10216 (Decin et al. 2010). The results obtained by Herschel seem to indicate that the clumpy structure of these circumstellar shells allow UV photons from the ISM to penetrate deep enough through the envelope and trigger a set of reactions leading to the production of water.

In addition, SPIRE-FTS spectroscopy of three carbon-rich evolved objects, AFGL 2688, AFGL 618 and NGC 7027 (Wesson et al. 2010) reveals a rich and complex molecular chemistry. From the more than 150 lines that can be observed in each object the physical conditions of their shells can be determined. It is worth to mention the detection of large velocity gradients in AFGL 618, the presence of water in AFGL 2688 for the first time, and the confirmation of its presence in AFGL 618 in both orto and para forms. In addition, the detection of the $J=1-0$ line of $\mathrm{CH}^{+}$in NGC 7027 is also reported.

The circumstellar shells of evolved stars are enormous molecular laboratories which allow very complex molecular chemistry; hundreds of lines are observed in some of these sources, sometimes complex organic molecules, considered to be the building blocks of life. In Fig. 3 we show, as an example, the rich molecular spectrum of the circumstellar shell around the red supergiant star VY CMa, obtained with PACS (Royer et al. 2010), an oxygen-rich star in an extreme evolutionary state that could explode as supernova at any time. The SPIRE spectrum (not shown) is dominated by prominent features coming from carbon monoxide $(\mathrm{CO})$ and water $\left(\mathrm{H}_{2} \mathrm{O}\right)$. Overall, more than 900 spectral lines are detected, with more than half of them belonging to water, indicating that chemical non-equilibrium processes determine the abundance fractions of the inner envelope.

HIFISTARS: (The physical and chemical properties of circumstellar environments around evolved stars; P.I. V. Bujarrabal) is a HIFI programme aiming at obtaining very 


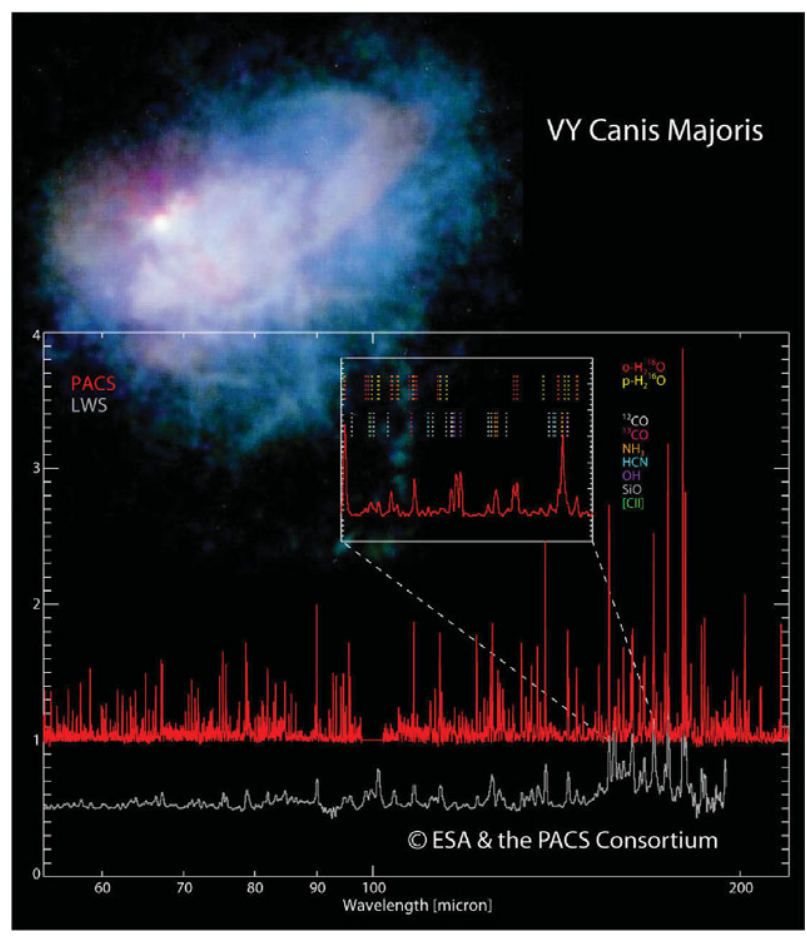

Figure 3. PACS spectrum of the red supergiant VY CMa between 57 and $210 \mu \mathrm{m}$. For comparison, depicted in gray, and offset by -0.5 , is the observation from the ISO Long Wavelength Spectrometer (LWS). The inset shows a zoom into the 156 to $172 \mu \mathrm{m}$, containing 44 different identified molecular lines (Royer et al. 2010).

high-resolution spectra of the environments around old stars. This allows the detection of common molecules, such as water and carbon monoxide, and provide information on the physical conditions and chemical composition of the material surrounding the stars. The high resolution of HIFI allows the velocity of the gas to be measured, providing information on the dynamics, structure, and evolution of the objects.

In particular, HIFI is able to obtain information on the warm components of these objects. The study of $\mathrm{CO}$ and $\mathrm{H}_{2} \mathrm{O}$ emission in some representative sources has allowed the determination of their physical conditions and molecular abundances in a variety of regimes. Noteworthy are the detection of intense $\mathrm{H}_{2} \mathrm{O}$ emission in O-rich, C-rich and Stype AGB stars, or the results obtained from the analysis of the $\mathrm{NH}_{3}$ emission in O-rich evolved stars, from which valuable information is deduced on the peculiar chemistry of this molecule (Menten et al. 2010).

Observed ${ }^{12} \mathrm{CO}$ and ${ }^{13} \mathrm{CO}$ profiles are compared with model predictions based on previously obtained ground-based and milimeter-wave data. Wide profiles showing sometimes spectacular line wings have been found and with HIFI we can study the excitation properties of the high-velocity emission, which is known to come from fast bipolar outflows. In some sources, like AFGL 618, the presence of relatively hot, recently shocked gas is derived (Bujarrabal et al. 2010). The fast bipolar outflow found in this source is much hotter than previously estimated; for instance, the gas flowing at $100 \mathrm{~km} \mathrm{~s}^{-1}$ must have a temperature higher than $\sim 200 \mathrm{~K}$. Probably, this very fast outflow, with a kinematic age $<100 \mathrm{yr}$, has been accelerated by a shock and has not yet cooled down. In contrast, the fast winds in $\mathrm{OH} 231.8+4.2$ and NGC 6302 are cold, with $T \sim 30 \mathrm{~K}$ (a warmer component 


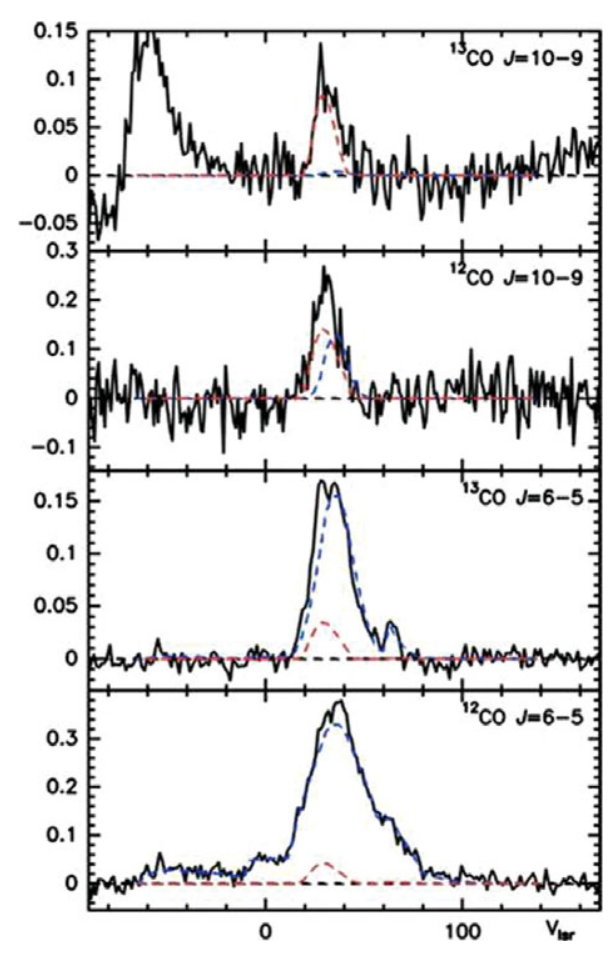

Figure 4. HIFI spectrum of $\mathrm{OH} 231.8+4.2$ where high-excitation, low velocity components are observed revealing the presence of a new warm emission component at $150 \mathrm{~K}$, which is needed to explain the whole set of molecular lines observed with Herschel (Santander-García et al. this proceedings).

has recently been found in $\mathrm{OH} 231.8+4.2$ at low velocity - see Fig. 4). Other nebulae, like CRL 2688, show intermediate temperatures, with characteristic values around $100 \mathrm{~K}$. Two-component models are sometimes needed to explain the complex structure of the nebulae observed. The differences in temperature in the different nebulae can be due to cooling after the gas acceleration (that is probably due to shocks); for instance, CRL 618 is a case of very recent acceleration, less than $\sim 100 \mathrm{yr}$ ago, while the fast gas in $\mathrm{OH} 231.8+4.2$ was accelerated $\sim 1000$ yr ago. We also find indications that the densest gas tends to be cooler, which may be explained by the expected increase of the radiative cooling efficiency with the density. Finally, for NGC 7027, a new model consisting of 3 nested, mildly bipolar shells, and a pair of high-velocity polar blobs results from the obervations made with Herschel, with the inner shell, located just beyond the PDR, showing low abundances, high density and a hot temperature $T>200 \mathrm{~K}$. In addition, its higher velocity is indicative of a front shock travelling outwards throughout the nebula, likely implied in the shaping of the nebula.

Regarding the observations of water made under HIFISTARS, it is worth mentioning the detection of water vapour toward the carbon star V Cyg (Neufeld et al. 2010), the second after the SWAS detection of water toward CW Leo $=$ IRC+10216. Emission from water has been further confirmed more recently in another eight carbon-rich stars (Neufeld ey al. 2011), whose origin is not yet well understood.

Other results obtained from Key Programmes other than MESS and HIFISTARS include the surprising detection of cold dust around SN1987A (Matsuura et al. 2011). The observations reveal the presence of a population of cold dust grains radiating with a 


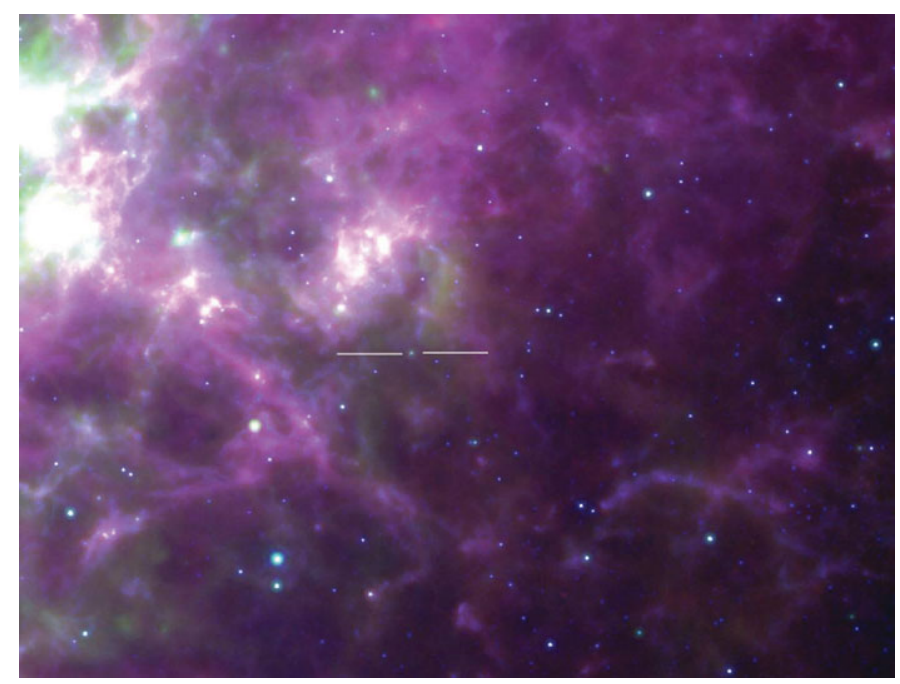

Figure 5. A Herschel $250 \mu \mathrm{m}$ and Spitzer/IRAC 8 and Spitzer/MIPS $24 \mu \mathrm{m}$ composite image of SN 1987A and the surrounding regions. The position of SN 1987A is indicated by two horizontal lines (Matsuura et al. 2011).

temperature of about 17 to $23 \mathrm{~K}$ at a rate of about 220 times the luminosity of the Sun. The intensity and spectral energy distribution of the emission suggest a dust mass of about 0.4 to 0.7 times the mass of the Sun. The radiation must originate from the supernova ejecta and requires the efficient precipitation of all refractory material into dust. The observations imply that supernovae could produce the large dust masses detected in young galaxies at very high redshifts.

\subsection{Cycle 1 Herschel programmes on evolved stars}

A total of 10 programmes addressing mostly spectroscopic studies on evolved stars and planetary nebulae were approved for execution in the Cycle 1 call for Guaranteed Time proposals for a total observing time of $110 \mathrm{~h}$, representing 20

A molecular line survey of IRC+10216 (P.I.: J. Cernicharo), a sub-milimeter catalogue of circumstellar envelopes of stars observed with HIFI in various high excitation CO lines (P.I.: D. Teyssier), a sensitive search for light hydrides towards IRC +10216 (P.I.: M. Agúndez), or a search for forsterite dust in the circumstellar ejecta of evolved stars at different evolutionary stages in their way to become planetary nebulae (P.I.: J. Blommaert) are a few examples of the kind of studies that were approved for execution in this call.

In addition, a total of 24 programmes, for a total observing time of $\sim 660 \mathrm{~h}$, representing around $10 \%$ of the total available time for the Cycle 1 Open Time call were also approved for execution, addressing a large variety of topics related to the field of evolved stars and planetary nebulae.

The largest one will map the far-infrared emission of a sample of planetary nebulae in various spectral lines (P.I.: T. Ueta), while others will analyse the far-infrared bands of PAHs in the Red Rectangle (P.I.: Ch. Joblin), the molecular complexity of the Orich pre-planetary nebula OH231.8+4.2 (P.I,: Sánchez-Contreras) and the massive red supergiant VY CMa (P.I. J. Cernicharo), the chemistry of water fountain stars (various programmes) or the evolutionary history of R Coronae Borealis stars (P.I.: G. Clayton).

Some follow-on studies will address the puzzle of water vapour in carbon-rich stars (P.I: D. Neufeld), and there will also be room for new projects addressing the analysis of the 
dust processing and grain growth in Keplerian discs around binary post-AGB stars (P.I.: C. Gielen) or the formation of fullerenes around evolved stars (P.I,: J. Bernard-Salas).

\subsection{Cycle 2 Herschel programmes on evolved stars}

At the time of the publication of these proceedings the results of the second and final call for proposals to observe with Herschel during the last year of the mission will have just been announced. The Guaranteed Time component of this call will contain followon studies e.g. on the faint extended dust envelopes, bow shocks and arcs found around AGB stars and young planetary nebulae (P.I.: N. Cox), the mapping of crystalline silicate forsterite emission in individual sources (various programmes) and additional molecular observations in an extended sample of AGB and post-AGB objects (P.I.; V. Bujarrabal), but also novel research studies that will hopefully cover areas not yet covered with previously approved programmes before Herschel runs out of helium in early 2013.

\section{4. ... and much more is yet to come}

A lot of people all around the world have been working in the last 20 years to make this mission possible. Thanks to all of them Herschel is now a fully working observatory with its three science instruments working at full speed, providing plenty of new food for thought every day. Not only are the observatory and the instruments working very well, but it is already clear that in this unexplored region of the spectrum the Universe is even more interesting that we ever thought. This is certainly only the beginning of what Herschel will be able to provide us in the coming two years. Herschel is undoubtedlt called to expand significantly our knowledge on the formation and evolution of stars and galaxies, showing us the Universe in we live as we had never seen it before.

\section{Acknowledgements}

Herschel is an ESA space observatory with science instruments provided by Europeanled Principal Investigator consortia and with important participation from NASA.

\section{References}

Bujarrabal, V., Alcolea, J., Soria-Ruiz, R., et al. 2010, A\& A, 521, L3

Cox, N. L. J., Kerschbaum, F., van Marle A. J., et al. 2012, A\&3A, 537, 35

Decin, L., Agúndez, M., Barlow, M. J., et al. 2010, Nature 467, 64

García-Lario, P. 2011, in: I.N. Evans, A. Acomazzi, D.J. Mink \& A.H. Rots (eds.), ADASS XX. ASP Conference Proceedings, 442, 225

Jorissen A., Mayer, A., van Eck, S., et al. 2011, A\&A A, 532, 135

Kerschbaum, F., Ladjal, D., Ottensamer, R., et al. 2010, A\&A, 518, L140

Kerschbaum, F., Mecina, M., Ottensamer, R., et al. 2011, AAP Conf. Series 445, Why Galaxies Care About AGB Stars II, ed. F. Kerschbaum \& T. Lebzelter

Ladjal, D., Barlow, M., Groenewegen, M. A. T., et al. 2010, A\&A 518, L141

Mayer, A., Jorissen, A., Kerschbaum, F., et al. 2011, $A \& A$, 531, L4

Matsuura, M., Dwek, E., Meixner, M., et al. 2011, Science 333, 1258

Menten, K. M., Wyrowsky, F., Alcolea, J., et al. 2010, A\&A 521, L7

Molinari, S., Swinyard, B., Bally, J., et al. 2010, A\&A, 518, L100

Neufeld, D., González-Alfonso, E., Melnick, G., et al. 2010, A\&A 521, L5

Neufeld, D., González-Alfonso, E., Melnick, G., et al. 2011, ApJ Letters 727, L29

Pilbratt, G. 2011, in: EAS Publication Series, 52, 5

Pilbratt, G., Riedinger, J. R., Passvogel, T., et al. 2010, A\&A A, 518, L1

Royer, P., Decin, L., Wesson, R., et al. 2010, A\& $A$ 518, L145

van Hoof, P. A. M., van de Steene, G. C., Barlow, M., et al. 2010, A\&AA 518, L137

Wesson, R., Cernicharo, J., Barlow, M. J., et al. 2010, A\&SA 518, L144 